\title{
Factores internos que intervienen en la planificación estratégica del proceso de admisión estudiantil
}

\author{
Internal factors involved in the strategic planning of the student \\ admission process
}

\author{
Ysamar Rodríguez \\ ysa.rodriguez.90@gmail.com \\ Código ORCID: 0000-0003-0366-9183 \\ Universidad del Zulia, Venezuela
}

Artículo recibido septiembre 2019 / Arbitrado en octubre 2019 / Publicado en enero 2020

\begin{abstract}
RESUMEN
El estudio identifico los factores internos que intervienen en la planificación estratégica aplicada en el proceso de admisión estudiantil. Metodológicamente se tipificó como descriptiva, con diseño no experimental, transeccional y de campo. La población quedó constituida por el personal encargado de la admisión estudiantil de las instituciones universitarias públicas ubicadas en la Costa Oriental del Lago. La técnica de recolección de datos utilizada fue la encuesta y el instrumento un cuestionario con escala Lickert, compuesto por 18 ítems, se utilizó el juicio expertos para la validez del mismo y el método de Alfa de Cronbach para su confiabilidad la cual arrojo un valor de 0,92. El análisis de los datos se calculó mediante la media aritmética. Se concluye que los factores internos: cultura organizacional, naturaleza de la tarea, grupo de trabajo, estilo de liderazgo, tecnología y sindicatos, permiten el modelaje de la estrategia de la organización.
\end{abstract}

Palabras clave: Cultura organizacional, estilo de liderazgo, factores internos, grupo de trabajo, naturaleza de la tarea, planificación estratégica, sindicatos, tecnología

\begin{abstract}
The study identified the internal factors that intervene in the strategic planning applied in the student admission process. Methodologically it was typified as descriptive, with a non-experimental, transectional and field design. The population was constituted by the personnel in charge of the student admission of the public university institutions located in the Eastern Coast of the Lake. The data collection technique used was the survey and the instrument a questionnaire with a Lickert scale, composed of 18 items, the expert judgment was used for its validity and the Cronbach's Alpha method for its reliability, which yielded a value of 0.92 . Data analysis was calculated using the arithmetic mean. It is concluded that the internal factors: organizational culture, nature of the task, work group, leadership style, technology and unions, allow the modeling of the organization's strategy.
\end{abstract}

Key words: Organizational culture, leadership style, internal factors, work group, nature of the task, strategic planning, unions, technology 


\section{INTRODUCCIÓN}

Ante el nuevo papel del conocimiento, las instituciones universitarias adquieren un papel protagónico, al ser el centro de mayor concentración de investigaciones y semillero del proceso de transferencia, por tanto se convierten en instrumentos fundamentales en las que se apoya la sociedad e impulsan un crecimiento de la ciencia y tecnología.

En este sentido, las instituciones universitarias han transitado un largo camino desde su creación, ofreciendo 0 al menos intentando, dar respuestas a las demandas del contexto histórico, político, económico y social a través del cual han evolucionado. Visto así, es función de estas instituciones ofrecerle al estudiantado oportunidades para que defina su campo de estudio y trabajo, brindarle una capacitación científica, humanística y técnica que le permita incorporarse al trabajo productivo y orientarlo para la prosecución de estudios de educación superior.

De manera que una política de las instituciones universitarias es la planificación, organización, ejecución y control de los procesos de admisión estudiantil. Bonucci (1997) citado por Blanco y otros (2010), define el sistema de admisión a los estudios de tercer nivel como un conjunto de procedimientos o medios que diseñan y utilizan las instituciones de Educación Superior para captar estudiantes que en sus estudios universitarios obtengan un rendimiento adecuado.

Dentro de este contexto, las instituciones universitarias se han visto en la necesidad de establecer un proceso de gestión para poder abordar la admisión estudiantil con una visión clara de crecimiento, que le permita incorporar al sistema a grupos socialmente heterogéneos que posean las aptitudes, motivación y vocación necesarias para hacer frente de forma exitosa a la dinámica de los estudios superiores.

Por ello, una política de admisión debe estar en función del modelo de universidad hacia donde deba dirigir sus esfuerzos, y de acuerdo con este modelo la planificación de la actividad universitaria juega un papel importante, tal como lo plantea Serna (2008), quien refiere que la planificación estratégica implica un proceso de dinamización emprendido en función de alcanzar los objetivos, centrados en el mejoramiento, optimización y elevación de la calidad educativa. Por su parte, Lepeley $(2003$, p.45) afirma "es el camino que la dirección de una institución elige para poner en práctica la misión de la organización utilizando los recursos humanos, físicos y financieros en la forma más efectiva y eficiente posible".

Estas posturas permiten evidenciar, la importancia de la planificación en las instituciones universitarias para impulsar el cambio de los escenarios con visión constructiva en sus procesos, lo cual requiere de un continuo análisis para seleccionar una dirección con personas eficaces que guíen el quehacer universitario mediante acciones viables para su desarrollo, escenarios factibles de lograr a través de la planificación estratégica, herramienta clave que permite, en palabras de Graffe $(2003$, p. 11) la transformación organizacional:

"Las empresas e instituciones
educativas para ser competitivas y
exitosas, y, ofrecer un servicio de
calidad y excelencia requieren planear
su cambio y llevado a adelante con el
esfuerzo de los hombres y mujeres que
trabajan en ellas (...) es decir los
docentes, gerentes y supervisores de la
gestión educativa. Si asume el cambio
de cada uno como un reto personal y
profesional, se logra la transformación


de la institución y esto, a su vez, logra cambios en el entorno".

Así las cosas, es preciso entender la importancia que debería tener para las universidades el contar con una planificación estratégica que proporcione los lineamientos específicos, concretos y claramente definidos, que la guíen de manera eficiente hacia la excelencia académica $y$, por ende, a la satisfacción de la demanda constante a las que están inmersas.

En referencia a las exposiciones anteriores, puede decirse que la planificación estratégica es un instrumento que sirve para direccionar el proceso de admisión estudiantil, permitiendo planificar con exactitud las actividades a realizar y de esta manera evitar improvisaciones, puesto que la misma tiene la finalidad de buscar las acciones pertinentes para definir un sistema que, atendiendo a la misión, visión, valores y planes de desarrollo de cada institución, dé una respuesta socialmente justa a la demanda de ingreso de los aspirantes a sus aulas.

En el caso específico de las instituciones universitarias públicas de la Costa Oriental del Lago, el proceso de admisión se centra en los fundamentos legales de la educación universitaria venezolana, cuyo fin principal es la inserción de jóvenes según sea el plan de crecimiento matricular de cada institución. El mismo, debe ser garante de la captación de nuevos ingresos a través de la promoción de los programas académicos, relacionándose éstos con los procesos de inscripción y todos los movimientos de permanencia, registro y control de la data académica de la población estudiantil de pregrado; de este modo cumplir con los procesos de culminación de estudios de los estudiantes que conlleven al solemne acto de grado, expedición de certificados, solvencias y solicitudes académicas.
Ahora bien, la nueva política implementada por el Ministerio del Poder Popular para la Educación Universitaria, de eliminar las pruebas de admisión de las universidades y asignar a todos los estudiantes a través de un sistema de la Oficina de Planificación del Sector Universitario (Opsu), implica una planificación que le permita asumir la propuesta del Ejecutivo Nacional de ingreso de los jóvenes en igualdad de condiciones y de oportunidades, sin discriminación sociales, étnicas o físicas; pero para ello, se requiere que con veracidad y objetividad se asignen los recursos necesarios y obligatorios en todos los ámbitos para poder dar respuesta a los nuevos aspirantes de las instituciones universitarias públicas.

Por lo antes expuesto, es necesario analizar la planificación estratégica, identificando las oportunidades y amenazas del afuera, como las fortalezas y las debilidades del adentro, articulando una visión, misión, metas y objetivos institucionales acordes con las expectativas de la comunidad, para desarrollar estrategias y tácticas que, en el marco de un plan, se orienten en satisfacer las necesidades de los nuevos ingresos y de las instituciones universitarias en general.

En este sentido, en las instituciones universitarias, el proceso de planificación estratégica se debe convertir en un proceso de aprendizaje sobre la organización, de tal manera que en la medida que ésta aprenda sobre sí misma, puede ella ir generando su propio mecanismo de planeación y conformando su propio destino. Debido a lo anteriormente expuesto, se plantea en este estudio la necesidad de identificar los factores internos que intervienen en la planificación estratégica aplicada en el proceso de admisión estudiantil de las instituciones universitarias públicas de la Costa Oriental del Lago. 


\section{MATERIALES Y METODO}

Metodológicamente se tipificó como descriptiva, con diseño no experimental, transeccional y de campo. La población quedó constituida por 12 secretarios docentes, 16 coordinadores académicos, 3 coordinadores docentes, y 20 personal administrativo, para un total de 51 sujetos encargados de la admisión estudiantil de las instituciones universitarias públicas ubicadas en la Costa Oriental del Lago, específicamente: La Universidad del Zulia Núcleo Costa Oriental del Lago, Universidad Nacional Experimental "Rafael María Baralt" y el Instituto de Tecnología de Cabimas.
La técnica de recolección de datos utilizada fue la encuesta y el instrumento un cuestionario con escala Lickert, compuesto por 18 ítems, se utilizó el juicio expertos para la validez del mismo y el método de Alfa de Cronbach para su confiabilidad la cual arrojo un valor de 0,92. El análisis de los datos se calculó mediante la media aritmética. Para tal efecto, la investigadora diseñó un baremo para su análisis, en el cual se muestra la alternativa de respuesta, intervalo y categoría asignada, tal como se muestra en el tabla 1.

Tabla 1. Baremo para la interpretación de la media aritmética

\begin{tabular}{lccc}
\multicolumn{1}{c}{ Alternativa } & Rango & Intervalo & Categoría \\
\hline Totalmente de Acuerdo (TA) & 5 & $4.21-5.00$ & Muy alta aplicación \\
De Acuerdo (Da) & 4 & $3.43-4.20$ & Alta aplicación \\
Indiferente (I) & 3 & $2.62-3.42$ & Moderada aplicación \\
Desacuerdo (D) & 2 & $1.81-2.61$ & Baja aplicación \\
Totalmente Desacuerdo (TD) & 1 & $1.00-1.80$ & Muy baja aplicación \\
\hline
\end{tabular}

Fuente: Rodríguez (2020)

\section{RESULTADOS Y DISCUSION}

En la tabla 2 se evidencia para el indicador cultura organizacional, un promedio de 4,59 indicando muy alta aplicación de un conjunto de percepciones, sentimientos, actitudes, hábitos, creencias, valores, tradiciones y formas de interacción dentro y entre los grupos existentes en los procesos de admisión estudiantil de las instituciones analizadas. 
Tabla 2. Cultura Organizacional

\begin{tabular}{|c|c|c|c|}
\hline \multirow[b]{3}{*}{ Ítems } & \multicolumn{2}{|l|}{1} & 3 \\
\hline & \multicolumn{3}{|c|}{$\begin{array}{l}\text { Usted como coordinador, secretario o personal de apoyo en los } \\
\text { procesos de admisión estudiantil, contribuye a: }\end{array}$} \\
\hline & $\begin{array}{l}\text { Compartir información } \\
\text { que permita determinar } \\
\text { el grado de adaptación } \\
\text { laboral. }\end{array}$ & $\begin{array}{l}\text { Difundir los valores que } \\
\text { permitan mejorar los } \\
\text { objetivos trazados en la } \\
\text { planificación } \\
\text { estratégica }\end{array}$ & $\begin{array}{l}\text { Mantener las conductas } \\
\text { gerenciales apropiadas } \\
\text { que sirven de soporte a } \\
\text { los principios básicos } \\
\text { de la institución. }\end{array}$ \\
\hline Promedio/ Ítems & 4,55 & 4,59 & 4,65 \\
\hline Categoría & Muy alta aplicación & Muy alta aplicación & Muy alta aplicación \\
\hline $\begin{array}{l}\text { Promedio/Indicador } \\
\text { Categoría }\end{array}$ & \multicolumn{3}{|c|}{$\begin{array}{l}\text { 4,59 } \\
\text { Muy alta aplicación }\end{array}$} \\
\hline
\end{tabular}

Fuente: Rodríguez (2020)

De esta manera, los resultados obtenidos se apoyan en lo expresado por Zapata (2007), quien define la cultura organizacional como un esquema de referencia para patrones de información, comportamiento y actitudes que compartimos con otros en el trabajo y que determinan el grado de adaptación laboral; en esta medida representan un aprendizaje continuo en el cual la cultura organizacional se enriquece con los aportes de los individuos, se perpetúa a través de ellos a la vez que las personas enriquecen sus entornos.

Asimismo, tiene alta coincidencia con lo expuesto por la investigadora, al considerar que la cultura organizacional determina la forma como funciona las instituciones objeto de estudio $y$, esta se observa a través de sus estrategias, formada de valores y normas permitiendo a cada uno de los individuos identificarse con ellos $y$, poseer conductas positivas dentro de la misma, obteniendo mayor productividad y demostrando al público una buena imagen del lugar donde laboran y/o estudian, y lo satisfecho que se siente en ella.

Seguidamente, en la tabla 3, se encuentran los resultados obtenidos para el indicador naturaleza de la tarea, donde se observa una media de 4,46 indicando muy alta aplicación de las actividades futuras, para adaptarse a los cambios y las demandas del entorno de acuerdo con la razón de ser de los procesos de admisión estudiantil de las instituciones bajo estudio. 
Tabla 3. Naturaleza de la tarea

\section{4}

Usted como coordinador, secretario o personal de apoyo en los procesos de admisión estudiantil, contribuye a:

Ítems

Utilizar la planificación estratégica para cumplir con la naturaleza de la tarea.

4,53

Muy alta aplicación

Muy alta aplicación

Llevar a cabo las actividades necesarias para

obtener un resultado previsto.

\section{4,51} 4,35

Categoría

Promedio/Indicador

Categoría

Fuente: Rodríguez (2020)

La situación mostrada, tiene muy alta coincidencia con lo expresado por Mondy y Noe (2005), al referir que tarea es el grado en el que un puesto incluye una unidad identificable de trabajo que se lleva a cabo de principio a fin, el conjunto de actividades a llevar a cabo por el trabajador para obtener un resultado previsto.

De igual manera, los resultados coinciden con el juicio de la investigadora, quien refiere que las tareas comprenden tanto contenidos como métodos de trabajo, asimismo conforman en conjunto una acción completa, las cuales se encuentran articuladas con las metas, objetivos además deben estar alineadas con las estrategias de la organización para llevar a cabo sus actividades internas, así como con los procesos productivos, la organización y empresariales.

En lo que respecta al indicador grupo de trabajo, se tiene una media de 4,58 , tal como se muestra en la tabla 4, indicando muy alta aplicación de un conjunto de personas con conocimientos y habilidades para cumplir las metas establecidas en los procesos de admisión estudiantil en las instituciones universitarias analizadas. Así las cosas, hay que consolidar los grupos de trabajo como herramienta fundamental para alcanzar los objetivos, manteniendo la comunicación en el personal dentro de la institución para cumplir con las metas planteadas. 
Tabla 4. Grupo de trabajo

\begin{tabular}{|c|c|c|c|}
\hline \multirow[b]{3}{*}{ Ítems } & 7 & 8 & 9 \\
\hline & \multicolumn{3}{|c|}{$\begin{array}{l}\text { Usted como coordinador, secretario o personal de apoyo en los } \\
\text { procesos de admisión estudiantil, contribuye a: }\end{array}$} \\
\hline & $\begin{array}{l}\text { Consolidar los } \\
\text { grupos de trabajo } \\
\text { como herramienta } \\
\text { fundamental para } \\
\text { alcanzar los } \\
\text { objetivos. }\end{array}$ & $\begin{array}{l}\text { Mantener la } \\
\text { comunicación } \\
\text { entre el personal } \\
\text { dentro de la } \\
\text { institución. }\end{array}$ & $\begin{array}{l}\text { Establecer grupos } \\
\text { de trabajo para } \\
\text { cumplir con las } \\
\text { metas planteadas.. }\end{array}$ \\
\hline Promedio/ Ítems & 4,57 & 4,65 & 4,51 \\
\hline Categoría & Muy alta aplicación & Muy alta aplicación & Muy alta aplicación \\
\hline Promedio/Indicador & & 4,58 & \\
\hline Categoría & & Muy alta aplicación & \\
\hline
\end{tabular}

Fuente: Rodríguez (2020)

Estos resultados validan lo expresado por Shemershom y otros (2008), quienes refieren la cohesión de grupo como condiciones emocionales, por cuanto desde la perspectiva instrumental está determinada por la sensación de unión, compañerismo y solidaridad basada en la dependencia, en función de una meta común. También el mismo autor, asegura que los empleados, subalternos o miembros, entienden que no hay ninguna mejora demasiada pequeña, ya que, con el paso del tiempo pueden hacer una diferencia importante y los equipos muy cohesionados suelen tener menos tensiones, hostilidad y malos entendidos que los grupos menos cohesionado.

Asimismo, los resultados obtenidos tienen coincidencia con el juicio de la investigadora, al considerar que los grupos de trabajo deben ser cohesionados y unidos, donde exista la comunicación y cooperación a fin de alcanzar la misión y visión, así como los objetivos y metas de la institución.

Ahora bien, en esta exposición de resultados, le corresponde el turno al indicador estilo $d$ liderazgo, cuyo resultado se aprecia en la tabla 5, alcanzando un promedio de 4,46, indicando muy alta aplicación de un conjunto de habilidades gerenciales que poseen quienes se encargan de los procesos de admisión estudiantil de las instituciones analizadas, para influir en la forma de ser o actuar de un grupo de trabajo determinado, haciendo que este equipo trabaje con entusiasmo hacia el logro de sus metas y objetivos. 
Tabla 5. Estilo de liderazgo

\begin{tabular}{|c|c|c|c|}
\hline \multirow[b]{3}{*}{ Ítems } & 10 & 11 & 12 \\
\hline & \multicolumn{3}{|c|}{$\begin{array}{l}\text { Usted como coordinador, secretario o personal de apoyo en los } \\
\text { procesos de admisión estudiantil, contribuye a: }\end{array}$} \\
\hline & $\begin{array}{l}\text { Capacitar a los } \\
\text { líderes para } \\
\text { cumplir sus } \\
\text { actividades en los } \\
\text { procesos. }\end{array}$ & $\begin{array}{l}\text { Formar y capacitar } \\
\text { continuamente al } \\
\text { personal en cuanto a } \\
\text { liderazgo gerencial } \\
\text { para lograr cumplir } \\
\text { los objetivos }\end{array}$ & $\begin{array}{l}\text { Desarrollar un } \\
\text { liderazgo } \\
\text { efectivo que } \\
\text { permita obtener } \\
\text { un óptimo } \\
\text { desempeño del } \\
\text { personal }\end{array}$ \\
\hline Promedio/ Ítems & 4,43 & 4,47 & 4,49 \\
\hline Categoría & Muy alta aplicación & Muy alta aplicación & Muy aplicación \\
\hline Promedio/Indicador & \multicolumn{3}{|c|}{4,46} \\
\hline Categoría & \multicolumn{3}{|c|}{ Muy alta aplicación } \\
\hline
\end{tabular}

Fuente: Rodríguez (2020)

Estos resultados validan lo expresado por Gelabert (2010), quien asevera que el estilo de liderazgo es la forma de relacionarse entre los directivos o superiores y sus colaboradores haciendo que éstos se sientan más participativos e interesados en asuntos de la empresa.

En este sentido, también concuerda con la investigadora, quien considera el estilo de liderazgo como la capacidad de influenciar a un grupo de personas para lograr un conjunto de metas y objetivos planteados, utilizando los recursos disponibles y obteniendo resultados eficientes o más de lo esperado.

El siguiente indicador que se analiza, en esta dimensión, es el denominado: tecnología. Los resultados relativos a él, se resumen en la tabla 6 , observándose un promedio de 4,33, indicando muy alta aplicación de un conjunto de conocimientos de base científica que permite aplicar soluciones técnicas a problemas prácticos de forma sistemática y racional en los procesos de admisión estudiantil en las instituciones bajo estudio.

Los valores alcanzados para el indicador validan lo expuesto por Sallenave (2009), quien plantea que la tecnología es una fuerza impulsora que desarrolla continuamente nuevos productos y servicios, nuevas formas de aplicación, nuevos mercados demandantes. Permite obtener más información, más rápidamente y de calidad. Educa constantemente sobre lo bueno, lo diferente, lo no satisfactorio y hace tomar decisiones con mayor rapidez pero con precisión. 
Tabla 6. Tecnología

\begin{tabular}{|c|c|c|c|}
\hline \multirow[b]{3}{*}{ Ítems } & 13 & 14 & 15 \\
\hline & \multicolumn{3}{|c|}{$\begin{array}{l}\text { Usted como coordinador, secretario o personal de apoyo en los procesos } \\
\text { de admisión estudiantil, contribuye a: }\end{array}$} \\
\hline & $\begin{array}{l}\text { Implementar el uso de } \\
\text { nuevos modelos de } \\
\text { tecnología en los } \\
\text { diferentes departamentos } \\
\text { para maximizar la } \\
\text { efectividad de la gestión. }\end{array}$ & $\begin{array}{l}\text { Aplicar } \\
\text { tecnologías en } \\
\text { función del } \\
\text { desarrollo } \\
\text { institucional }\end{array}$ & $\begin{array}{l}\text { Dar a conocer } \\
\text { los avances } \\
\text { tecnológicos a } \\
\text { través de cursos } \\
\text { de formación, } \\
\text { charlas y otros. }\end{array}$ \\
\hline Promedio/ Ítems & 4,33 & 4,37 & 4,27 \\
\hline Categoría & Muy alta aplicación & Muy alta aplicación & Muy alta aplicación \\
\hline Promedio/Indicador & \multicolumn{3}{|c|}{4,33} \\
\hline Categoría & \multicolumn{3}{|c|}{ Muy alta aplicación } \\
\hline
\end{tabular}

Fuente: Rodríguez (2020)

Así mismo, para la investigadora las tecnologías pueden constituirse en amenazas graves si las instituciones han dejado de actualizarse, dejando de adquirir e implementar las tecnologías que les permitan mantener el proceso de admisión estudiantil competitivo.

Para finalizar el análisis de los indicadores utilizados en la dimensión factores internos que intervienen en la planificación estratégica, se tiene lo referente al indicador sindicatos. Para ello se muestra la tabla 7, contentiva de los hallazgos resultantes de la aplicación del instrumento de recolección de datos para el indicador en cuestión. Se aprecia entonces, que el indicador alcanza un promedio de 4,17, indicando alta aplicación de las acciones que ejercen las asociaciones permanentes de trabajadores cuya finalidad es defenderlos $y$ negociar con quienes gerencian los procesos de admisión estudiantil las condiciones laborales de las instituciones bajo estudio.

En este sentido, los resultados anteriores se sustentan en lo expresado por Alegría (2007) quien plantea que el sindicato es el instrumento creado por los trabajadores y protegido por la ley para asumir la defensa de sus intereses, tanto los específicos y derivados de la relación de trabajo, así como aquellos de naturaleza general propios de su condición humana, constituyendo una estructura organizada, reglamentada y con dinámica interna que le permite con propiedad que se hable de su creación, de su naturaleza jurídica, de su condición de sujeto capaz de asumir derechos, deberes y obligaciones, a través del ejercicio de la autonomía colectiva, cuya constitución se somete al principio de legalidad sindical, y forma parte del contenido de la libertad sindical. 
Tabla 7. Indicador: Sindicatos

\begin{tabular}{|c|c|c|c|}
\hline \multirow[b]{3}{*}{ Ítems } & 16 & 17 & 18 \\
\hline & \multicolumn{3}{|c|}{$\begin{array}{l}\text { Usted como coordinador, secretario o personal de apoyo en los procesos } \\
\text { de admisión estudiantil, contribuye a: }\end{array}$} \\
\hline & $\begin{array}{l}\text { Negociar con los } \\
\text { sindicatos el tiempo } \\
\text { de trabajo durante } \\
\text { los procesos de } \\
\text { admisión. }\end{array}$ & $\begin{array}{l}\text { Establecer } \\
\text { estrategias } \\
\text { patrono-empleado } \\
\text { en pro de los } \\
\text { procesos de } \\
\text { admisión. }\end{array}$ & $\begin{array}{l}\text { Tomar en cuenta } \\
\text { los sindicatos como } \\
\text { una herramienta } \\
\text { para mejorar las } \\
\text { condiciones del } \\
\text { trabajador. }\end{array}$ \\
\hline Promedio/ Ítems & 3,94 & 4,25 & 4,31 \\
\hline Categoría & Alta aplicación & Muy alta aplicación & Muy alta aplicación \\
\hline Promedio/Indicador & \multicolumn{3}{|c|}{4,17} \\
\hline Categoría & \multicolumn{3}{|c|}{ Alta aplicación } \\
\hline
\end{tabular}

Fuente: Rodríguez (2020)

Al mismo tiempo tiene alta congruencia con lo expuesto por la investigadora, al considerar que los sindicatos son importantes para el mejoramiento y protección de los intereses económicos y sociales de sus integrantes. Además, velan por los aspectos de representatividad de lo contractual, de la Convención Colectiva y todo lo concerniente a lo que es la norma laboral.

Ya analizados cada uno de los indicadores considerados en la dimensión denominada factores internos que intervienen en la planificación estratégica, se pasa a verificar los resultados arrojados para esta dimensión. En la tabla 8 se observa un promedio de 4,43 indicando muy alta aplicación de un conjunto de elementos que permiten cumplir las metas establecidas en los procesos de admisión estudiantil de las instituciones bajo estudio.

Los resultados de muy alta aplicación de la dimensión, validan lo expuesto por Chiavenato (2006), para quien el análisis de los factores internos es el estudio de los elementos claves que en su momento han condicionado el desempeño pasado, la evaluación de este desempeño y la identificación de las fortalezas y debilidades que presenta la organización en su funcionamiento y operación en relación con la misión; este análisis comprende aspectos tales como su talento humano, tecnología, estructura formal, redes de comunicación formales e informales, capacidad financiera, aspectos administrativos y operativos, entre otros. 
Ysamar Rodríguez

Tabla 8. Dimensión: Factores internos

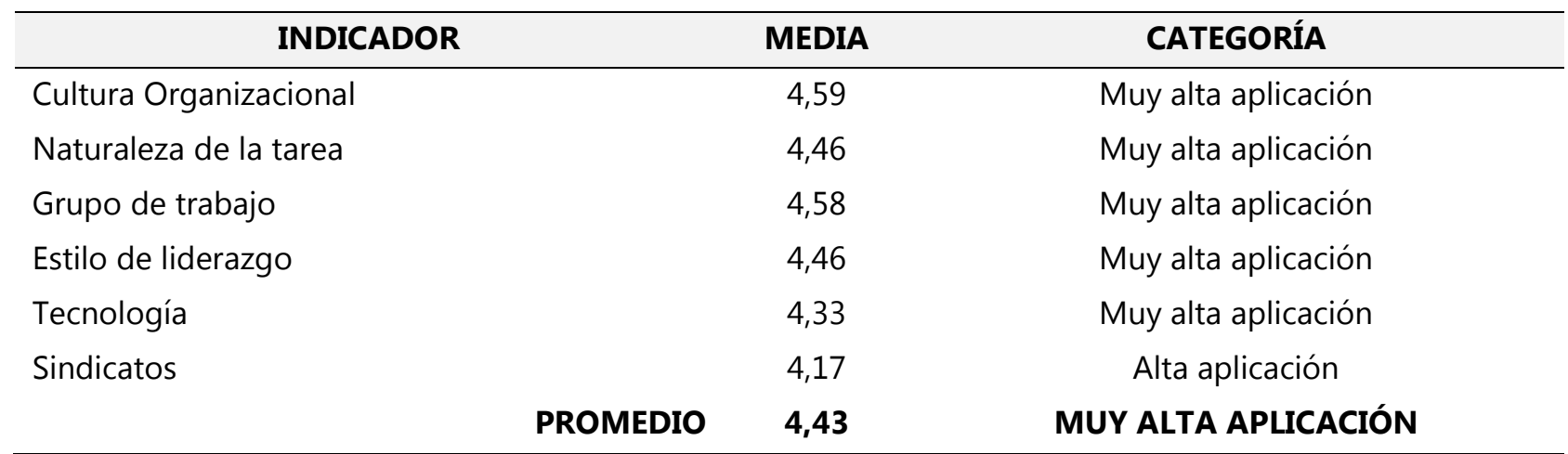

Fuente: Rodríguez (2020)

De igual manera, se valida lo expuesto por la investigadora, al considerar los factores internos como elementos cuya génesis se asocia a las actividades internas de cada organización, por lo tanto, los gerentes en las mismas, tienen la posibilidad de establecer políticas y planes de acción para obtener el mayor beneficio en coherencia con la finalidad de las instituciones universitarias públicas de la Costa Oriental del Lago

\section{CONCLUSIONES}

Con respecto al objetivo identificar los factores internos que intervienen en la planificación estratégica aplicada en el proceso de admisión estudiantil de las instituciones universitarias públicas de la Costa Oriental del Lago, se concluye que los factores internos, intervienen de tal manera que permiten el modelaje de la estrategia de la organización, por lo tanto, las estrategias de las mismas instituciones pueden ser diferenciables pues provienen de factores diferentes. Estos factores internos son los controlables dado que surgen de las áreas funcionales.

\section{REFERENCIAS}

Alegría, M. (2007) Derecho colectivo del trabajo, sindicatos, conflictos, negociación, convenios y seguridad social. Editorial El Nacional. Venezuela

Blanco, F; Flórez, E; Giménez, C. (2010).La equidad y la calidad en los procesos de admisión a la Educación Superior: Universidad Simón Bolívar y Universidad Central de Venezuela Revista de Pedagogía, vol. XXXI, núm. 89, julio-diciembre, 2010, pp. 251-276 Universidad Central de Venezuela Caracas, Venezuela

Bonucci, R. (1997). ¿Qué variables predicen el rendimiento universitario de los estudiantes? Estudio de un caso: La Facultad de Ingeniería en la ULA. Ponencia presentada en julio de 1997 durante el III Simposio sobre Políticas Educativas de Admisión en Educación Superior. Secretaría de la Universidad Central de Venezuela, Caracas

Bucheli, V. y González, F. (2007). Herramienta informática para vigilancia tecnológica VIGTECH. Revista Avances en Sistemas e Informática, 4(1), 117- 126

Chiavenato, I. (2001). Administración: teoría, proceso y práctica. Tercera edición. Mc. Graw Hill. Colombia 
Chiavenato, I. (2006). Administración de recursos humanos. Tercera edición. Editorial Mc. Graw Hill. México

David, F. (2010) Gestión Estratégica: Conceptos y Casos. Décima tercera edición. Pearson Prentice Hall. México

Gelabert, M. (2010) Gestión de personas: Manual para la gestión del capital humano en las organizaciones. 4ta Edición. Editorial ESCI. Madrid

Graffe, G. (2003) La Planificación y la Gerencia del cambio para la construcción de una Escuela Innovadora. Secretaria UCV. Venezuela

Ivancevich, J. (2004) Administración de Recursos Humanos. Editorial McGraw Hill. México

Jones, G. y George, J. (2006). Administración Contemporánea. México. Mc. Graw. Hill

Kinicky, A. y Kreitner, R. (2005) Comportamiento de las organizaciones. Mc Graw-Hill, Madrid, España

Lepeley, H (2003). Gestión y calidad en educación. Un modelo de evaluación. Editorial Mc Graw Hill. Chile

Machicado, J. (2010) Sindicato y Sindicalismo. Editorial New Life. Bolivia

Mondy, W y Noe, R (2005)- Administración de Recursos Humanos. Editorial Prentice Hall. México

Nube, S. y Sánchez, M. (2009). La Gerencia de la Calidad Educativa. CANDIDUS. Editores Educativos. Acarigua. Venezuela
Palomo, M. (2010). Liderazgo y motivación de equipo de trabajo. Editorial ESIC. Madrid

Sallenave, J. (2009) Gerencia y Planeación Estratégica. Tercera edición. Editorial Norma. Bogotá, Colombia

Sallenave, J. (2009) Gerencia y Planeación Estratégica. Tercera edición. Editorial Norma. Bogotá, Colombia

Serna, H. (2008). Gerencia estratégica. Décima edición. Editorial $3 r$ Editores. Bogotá, Colombia

Shemershom, J.; Hunt, J.; y Osborn, N. (2008) . Comportamiento organizacional. Limusa Wiley. Mexico

Stoner, R. (2002). Comportamiento organizacional. Mc.Graw Hill. México

Thompsom, A y Strickland, A. (2010). Administración estratégica. Decimoctava edición. Mc. Graw Hill. México

Villasmil, H. (2003) Fundamentos de derecho sindical venezolano. Venezuela. Editorial Texto C.A

Werther, W. y Davis, K. (2008). Administración de Personal y Recursos Humanos. Editorial Mc Graw-Hill Interamericana Editores, S.A. de C.V. México

Zapata, A. (2007). Cultura Organizacional. Ediciones Universidad del Valle. Cali, Colombia 